\title{
American Society of Hematology 2020 Podcast Collection: FL and DLBCL
}

\author{
Christopher Paul Fox $\cdot$ Wendy Osborne
}

Received: February 17, 2021 / Accepted: February 18, 2021 / Published online: March 9, 2021

(C) The Author(s) 2021

\section{PODCAST TRANSCRIPT}

Victoria Glasson (VG): Managing Editor of Advances in Therapy

Chris Fox (CF): Consultant hematologist, Nottingham University Hospitals NHS Trust

Wendy Osbourne (WO): Consultant hematologist, Freeman Hospital, Newcastle upon Tyne Hospital NHS Foundation Trust

VG: Hello and welcome to the Adis Rapid+ podcast series. We are bringing you a selection of podcasts covering the American Society of Hematology (ASH) 2020 conference, discussing the highlights of the data released. Today's podcast will be focusing on follicular lymphoma (FL) and diffuse large B cell lymphoma (DLBCL), focused on CAR (chimeric antigen receptor)-T cell therapies and the data presented at the ASH conference.

Speaking to us today is Dr. Christopher Paul Fox, consultant hematologist at Nottingham

Supplementary Information The online version contains supplementary material available at https:// doi.org/10.1007/s12325-021-01679-w.

C. P. Fox $(\bowtie)$

Nottingham University Hospitals NHS Trust, Nottingham, UK

e-mail: christopher.fox@nhs.net

W. Osborne

Freeman Hospital and Newcastle upon Tyne

Hospital NHS Foundation Trust, Newcastle, UK
University Hospitals NHS Trust, honorary clinical associate professor at the University of Nottingham, and also chair of the Aggressive Lymphoma Group of the NCRI (National Cancer Research Institute). He is joined today by Dr. Wendy Osborne, consultant hematologist at the Freeman Hospital, Newcastle upon Tyne Hospital NHS Foundation Trust, and a fellow member of the NCRI clinical subgroups looking at high-grade, Hodgkin, and T cell lymphomas.

Thank you so much for joining us today, Chris and Wendy. You are going to take us through your top highlights of the $\mathrm{FL}$ and DLBCL data that was released at the ASH conference and the implications of this data on future developments. A lot of important research was released at the conference, and we're going to focus mainly on CAR-T cell therapies.

CF: Thank you, Victoria, for the introduction and the opportunity to talk about some really interesting data on the CAR-T cell therapies that we saw presented at the ASH 2020 meeting, which is obviously a virtual meeting this year. But it worked really well.

It's a pleasure to have my colleague from Newcastle, Dr. Wendy Osborne, with me this evening. Wendy has, just to embarrass her slightly, she has a wealth of experience in both the delivery of CAR-T cell therapy and also in ongoing early phase trials, one of which we will 
talk about in the coming piece. So evening, Wendy, and nice to see you.

I wanted just to start maybe by setting the scene in terms of what we have available in the UK, in terms of CAR-T cell product. And you've had experience over the last couple of years, up in Newcastle, and where you think we're up to and perhaps some of the limitations of the existing proven, funded CD19 CAR-T products for DLBCL.

WO: At present in the UK, we are able to give the two products that are currently licensed. So both Axi-Cel and Tisagen, and it's for patients who have failed two prior lines of therapy. In the UK, they have to have fulfilled quite strict criteria in terms of previous therapy, and this is reviewed each week at a weekly panel to ensure that they have met the criteria before approval is given. And so we can now give both of these products in this setting, and for relapsed refractory primary mediastinal, we can also give the Axi-Cel product, because these patients were included in the trial. So at present, we have two, but obviously we have others in a clinical trial setting, and I'm hoping that in the future, we'll have CAR-Ts available for other indications.

CF: That's where I think the field is getting interesting and perhaps we could start by discussing some of the follicular lymphoma data that we saw at ASH. So as you say, the current approvals in funding is for the aggressive B cell lymphomas, but we're now starting to see emerging data in low-grade B-cell NHL (nonHodgkin lymphoma) and of course follicular is the commonest of those diseases. What were your impressions for the two main data sets that we saw with follicular lymphoma and CD19 CAR-T therapy?

WO: I think they look very encouraging. ZUMA-5 data were presented as well as the Elara trial with the Tisagen data $[1,2]$, and they were pretty similar in terms of the patient groups; so it was patients with follicular lymphoma who'd failed two prior lines of therapy. In the Elara trial, they'd had to have relapsed within 6 months of their most recent therapy, so maybe a slightly different group [2], but the efficacy looked good. The numbers in the trial looked good, particularly for ZUMA-5, with over
140 patients had been infused [1]. The response rates, again, encouraging, over $70 \%$. So this was encouraging data for a group of patients who, it's slightly different from aggressive lymphoma because we do still have treatment options for these patients, and we do have to consider where this will fit in our treatment pathway, because this group are heterogeneous. We have the patients who relapse early, many of whom could have gone on and actually transformed, and the outcomes can be poor. But we also have patients who relapse after two lines but they've had a good duration of their maybe first CR (complete response).

And in those patients, we've got to think carefully about whether using a potentially toxic treatment is going to be in their best interest. So I'm going to be interested in longer follow-up for this patient group and I think we're going to be discussing, obviously, there were loads of data with bispecifics in follicular as well, about which approach is going to be the optimal for our patients. I don't know. What did you think, Chris?

CF: I think there are really good points. Like you, I was really impressed by the response rates, the CR rates were $60 \%, 70 \%$ and comparable across the two studies. The toxicity, to me, seemed lower than perhaps we see in the aggressive lymphomas. That might be a function of the disease and the microenvironment associated.

I think that the most important thing to remember for us clinicians who treat follicular lymphoma is that most of our patients do very well with standard therapy. There is this really difficult group, the so-called POD24s, perhaps half of whom, as you say, are aggressive transformations. But nonetheless, there's a group of difficult low-grade follicular lymphomas, who I think this therapy is probably where the attention should be.

I think the question about how CAR-T cell therapy may fit in the paradigm for follicular lymphoma alongside the bispecific antibodies, I think that's really tricky, and I think that there's relative merits for both of those technologies, and I think it's something to really, really watch over the next couple of years. 
Do you have any thoughts particularly on the toxicity issue with the folliculars? Do you think it's a disease-related phenomena? Or how do you interpret that side of everything?

WO: So we know that if we're putting our patients with high-grade disease with a lot of bulk, then they're more likely to get CRS (cytokine release syndrome) and neurotoxicity. Certainly our practice now is to quite aggressively debulk patients during the bridging time, so that we are taking them into CAR-T when they're aggressive lymphoma patients to try and minimize that toxicity.

And you would hope, because like you I was quite impressed with the safety data, and you would hope that if it's a lower-grade disease maybe with less bulky disease, that there would be associated less toxicity.

I think what's going to also be interesting within this group is to really look at how their prior lines of therapy is going to affect the manufacturer of the products. Because many of the patients will have bendamustine. They would have had Tcell-depleting treatment, which many of our high grades won't have had. So when we would use bendamustine front line for the majority of our follicular lymphoma patients, will that practice change if we can see that that has a negative impact on our ability to obtain adequate T cell when we do leukapheresis? So exciting, but I do want to see more data.

CF: That's a really good point to start with, the thinking about $\mathrm{T}$ cell preservation, as it were, in the earlier lines of therapy and particularly in follicular lymphoma. So I think that's something that we really need to keep an eye on.

Let's chat now a bit more about the highgrade space, diffuse large B cell lymphoma. We've had the experience in clinical practice. We have promising data. Some patients are really benefiting, but there are limitations, both in terms of efficacy and toxicity. I think we saw some really interesting approaches from a number of abstracts at the ASH meeting looking at, first of all, dual antigen targeting. So rather than just designing your CAR construct targeting CD19, there are a couple of abstracts that caught my eye looking at different ways of targeting two antigens at the same time.
Probably wise to start with the study that you've been directly involved with as an investigator, the Alexander, AUTO3 study. Perhaps just describe the design of the study for people who are not familiar, and then we can discuss some of the other aspects.

WO: The Alexander study is using a bicistronic CAR, so that's using a single viral vector to produce a dual antigen targeting CAR for CD19 and CD20 [3]. And within the trial design, it's also in the later phase of the study, introduced a checkpoint inhibitor, so pembrolizumab. For safety reasons, that was initially brought in at day 14, and then when there were no data safety concerns; it's now delivered on day minus 1 of the study. So trying to look as if we can enhance that immune response by bringing in checkpoint blockade.

And having put patients into this study, it certainly seems a safe product. We saw minimal CRS and the neurotoxicity data are really encouraging. Because of this, the expanded phase of the study is now delivered as an outpatient-based approach. So at ASH this year, data were presented on, I think, about 49 patients, 17 of whom had had the product as an outpatient-based approach. And the CR rates were good, so $51 \%$, and more than $70 \%$ in the patients that had more than the 450 million dose.

So it looks as if the efficacy is good. But importantly, the toxicity profile is encouraging. Early days, we need more data, more patients in the study, but it certainly looks as if this approach may be of benefit.

I think that the future, what we've got to try and do is to get not only safety, efficacy... we also need to try and improve this turnaround time as well. Because it's the bridging time where patients are getting sick. And if I'm to keep them well for that month, that's a problem. I think that you could see this theme coming through at ASH this year with all of these approaches.

CF: I was also interested in the Alexander data set to see a kind of a dose response, really, in terms of the number of CAR-Ts returned. It was obviously a cautious step-up dosing, because of the potential concerns about the checkpoint inhibition. But that didn't really 
come through. And in fact, the toxicity profile looks potentially to be less than-it's difficult to compare across studies-but it looked to be probably less than some of the conventional products that we use. And that was a surprise to me. But is there any way to explain that? Or is it just we're better at managing the toxicity? Or is it truly less toxic?

WO: I don't think it's because we're better at managing it. Because if you look to the numbers of patients that actually even developed the CRS, the levels were low. So it was not like we were just giving a lot more tocilizumab and managing it more aggressively. We just did not see the toxicity with this product. So I think it's because of the way that the combination of the co-stimulatory design, domain design, and that is how the safety appears so encouraging.

I have to say I was surprised. Because obviously when we opened the study, we were nervous about bringing in a checkpoint inhibitor to the addition of a CAR-T. But it's because of the safety that now we are enrolling in this outpatient-based setting. So I think that this-I mean, I know I'm involved in this study-but I do think that this is one to watch.

CF: I think that as you say, a really key factor in the application of CAR-T cell therapy for high-grade disease, particularly, is this turnaround time issue. And the other interesting abstract at ASH was the data, the early phase small study, that Peter Borchmann presented [4]. Again, using a CAR-T product that targeted two antigens. But this was a fresh-to-fresh production. So 14-day turnaround time, perhaps compared to double that in a standard setting. Did you have any thoughts on the Borchmann data set? Anything that struck you particularly from that particular trial?

WO: Well, it was fantastic to see that this could be done within 14 days, and not having to freeze the product and being able to sort of deliver and turn around in 14 days.

And this makes it feel a lot more off the shelf. And that's always been the negative comments about CAR-T approaches, is that it's this having to wait, having to keep the patient well. But if we're able to just hold a patient for 14 days, then I'm encouraged.
I was impressed with the response data as well. Only, I think, it was about 12 patients were dosed. But five of them obtained a CR. And they were an older, a so-called AUTO-not-fit group of patients. So I was impressed. Small numbers, but (a) the fact that it can be delivered this way, and (b) the efficacy with the small numbers-it looks exciting.

CF: The age is really important. The median age 72 , considered not to be fit for a standard high dose autonomous transplant approach. So these patients would have generally been excluded from most CAR-T trials. So delivering what appears to be quite quick and safe products to older patients was a really key message from that abstract, I agree.

And then the third abstract that struck me in terms of targeting more than one antigen was a rather brave study, I thought, from the Chinese group. Where they took a really difficult group of patients with DLBCL, many of whom had stable disease or progressive disease. So clearly not chemosensitive at all at the time of enrolment [5]. And then instead of using the standard leukodepletion conditioning with the vidarabine cyclophosphamide, they used the standard BEAM conditioning that we would use in autologous transplant, having already collected regenerative stem cells, having also collected the T cells for the construct. A quite ambitious study to delivering a high dose therapy approach, returning the progenitor stem cells, and then within the first few days following this, putting back the CAR-T product.

I wasn't sure what to expect with the results they presented, and was quite impressed. I don't know if you were similarly or have any comments on that study?

WO: I think that this is one of the surprises, really, from ASH, because it's a totally different approach from anything that we've certainly done. But there's a little bit of me that thinks, well, it does make some sense.

We're now trying to debulk our patients, as I said. And we know that if we put patients in with lower metabolic tumour volume, the outcomes are good. You don't get stronger debulking therapy than giving a LEAM or a BEAM conditioning. 
So I thought it was a very brave study, because these patients are pretty sick. We all take patients through our BEAM orders regularly and they're pretty sick. Then to put in two separate CARs were delivered, so a CD19 and then a CD22. Yet the responses were phenomenal. I think it is over $80 \%$ or something CR rate for a high-risk group of people.

A very different approach. But one, again, I would be looking at. And I think we're learning more and more about the best ways to condition our patients, the best constructs to use. And things are changing. I think that we need to just keep looking at these different approaches so that we can try and pull out where we should take this forward in the future.

CF: As you say, clearly rational to debulk, both in terms of efficacy, but also in terms of toxicity. And actually the rates of CRS and neurotoxicity were quite low. Perhaps that is a function of the effectively myeloablative conditioning before the CAR-T.

And this was also a fresh-to-fresh production. As you say, it was a CAR-T cocktail of two different CARs rather than a bicistronic or like were used the Alexander study. So the platform could be really important to the conditioning, the antigen targeting, perhaps adjunctive therapy, like checkpoint inhibitors and I think we've got quite a lot of work to do, haven't we, to work out the best approach for individual patients. But of course, most of these data or all of these data that we've seen in DLBCL has been in the relapsed refractory setting; really difficult patients with limited options and some quite promising results.

But I think, for the first time, or certainly the first data I've seen in a prospective trial, looking at the use of CD19 CAR-T therapy in the firstline setting in DLBCL. And the ZUMA-12 study early data presented by Neelapu and colleagues was quite ambitious [6]. So just to briefly summarize, we had a group of patients with highrisk DLBCL at baseline, based on either an IPI (International Prognostic Index) score of 3 or more, or with adverse FISH (fluorescence in situ hybridization) features, so a double hit, making BCL2 rearrangement for example. They were given standard therapy with two cycles of R-CHOP. And then they had an early interim
PET scan, and if the early interim PET scan was positive, so Deauville 4 or more, then that second risk assessment point and so-called dynamic risk assessment prompted a change of therapy to CAR-T cell therapy. And I thought an ambitious approach; a nice idea to try and use a dynamic risk assessment to intervene on these patients we know would do badly with standard therapy. Interested in your thoughts on that ZUMA-12 study, quite a step forward really in how we're using CAR-T.

WO: I agree that we know that we only cure probably $60 \%$ of patients up front. And when they relapse, they're a lot harder to get back into a remission. So there's a lot of sense behind trying to pick out those high-risk patients and try and intensify that treatment up front. I admire that in terms of the design. I think that I'm just a little bit cautious about the selection of the high-risk patients. They were double hit but we don't know whether they were double hit with the immunoglobulin partner gene, which is now recognized as the highest risk group, and following the data from the Lunenburg Consortium. So I would certainly want to review that when we look at the outcomes. Because if they didn't have that partner gene, then we're basically not selecting out a high-risk group. And then the dynamic assessment, looking at Deauville 4 patients. Because we know that a lot of the Deauville 4 patients, it's not a true positive; we do see false positive PETs. And the positive predictive value after two cycles in some data sets suggest it's actually more accurate done after four. So I think that the data are intriguing. There's only a small number of patients. And the responses were better than you would think, obtaining a CR in the truly high-risk group. I think that I really want to look at the sub-analysis of how that is defined. But applauding a new novel design for trying to get a better outcome up front for patients.

CF: I think that's rather some important points there. So it's really important to scrutinize the risk profile of the patients entering, because as you say, many will do well with R-CHOP alone. But I think, if I recall, it was approximately $70 \%$ of patients were enrolled based on their IPI score alone, so 3 or more. 
But I didn't see a further breakdown of that 3 or more group. So that means 30\% were enrolled based on their side genetics or FISH results, which as you say, it's really crucial to know the immunoglobulin partner gene for the MYC rearrangement, because as you say, the Lunenburg data really clarified that for us. So I completely agree with you on that point. And secondly, I think that you're quite right; the role of PETs and how the PET data is interpreted is also really crucial. We've actually moved forward a lot with PETs, haven't we? And just using a Deauville score at a single time point is useful, but it's imperfect. And I think we're moving towards better PET metrics: TMTV (total metabolic tumour volume), delta SUV (standardized uptake value). I think they are probably smarter ways to use PETs dynamically.

So I think that that needs a bit more scrutiny, I think, as well. The last point I think I would make on the ZUMA-12 study is that, again, coming back to this issue of the turnaround time for CAR-T cell production. So I think it was 28 days in the study. So patients with high-risk DLBCL, you've identified is not responding optimally to first-line treatment. And then waiting, really, a month for an intervention. And you just wonder whether patients might deteriorate during that time, or whether there's a negative aspect to that gap in treatment. So I think that's again where fresh-to-fresh or a quicker product delivery could be quite important.

But I think nice to see that we're doing something about risk, both baseline and dynamically. And I think that's probably where the first-line field needs to move, and in a broader context.

WO: I agree.

CF: So I think we've talked about a lot of different approaches to CAR-T therapy. There are really exciting data. And I think we've still got a lot to learn.

I don't know if you could see any of these data impacting clinical practice in the near future, or how do you see the kind of field emerging in our everyday practice in the next 12-24 months, should we say?

WO: I think that the data for me, follicular CAR-T trials, I think that possibly could impact practice. I mean, they've got good numbers there and they've got good responses. And whether this becomes an available option for patients in the future, in terms of licensing, I'm not sure.

But I think that that's certainly something possible. And really I think the data from the phase 2 trials, just showing that we can do better in terms of efficacy. I thought it was really interesting how there was a big focus on not only efficacy this time but also on the toxicity and really trying to move to more outpatientbased approach. Recognizing that we've got two products which we're using now with good outcomes for many patients. But it does require patients to be in hospital for a minimum, usually, of 10 days when it's first delivered. And then to be close by to the hospital for the first month.

So if we can improve the safety, then it just opens up this treatment for other patients. And I think that we're going to be discussing, there was a huge amount of debate at ASH about bispecific versus CAR-T. And I think that we are going to be comparing these two approaches. And it's going to be really interesting to see which one is taken up, really, as our preferred treatment option for these patients in the future.

CF: Really exciting times with big focus on immunotherapy and T cells. And I think this is clearly going to make a big difference for our patients. And it's something that's great to be involved with.

Thank you for your time discussing it. I think we'll stop there and hopefully have another opportunity at the next conference.

WO: Lovely, thank you, Chris.

VG: Thank you both. That was such a fantastic roundup of all the data from ASH. So thank you so much for joining us today. And I really hope that this has been helpful to all of our listeners to give a great overview and summary of the ASH conference data. Please look out for our other podcast that we are releasing in the ASH 2020 coverage collection.

You can listen to more podcasts by subscribing to Adis Rapid+ podcast with your preferred podcast provider, or by visiting the website. A full list of declarations, including funding and author 
disclosure statements, can also be found on the journal website.

\section{DIGITAL FEATURES}

This article is published with digital features, including a podcast video and audio file, to facilitate understanding of the article. To view digital features for this article go to https://doi. org/10.6084/m9.figshare.14046725.

\section{ACKNOWLEDGEMENTS}

Funding. This podcast has been developed independently through an unrestricted educational grant by Novartis. The journal's Rapid Service and Open Access fee was funded by an unrestricted educational grant by Novartis.

Authorship. All named authors meet the International Committee of Medical Journal Editors (ICMJE) criteria for authorship for this article, take responsibility for the integrity of the work as a whole, and have given their approval for this version to be published.

Disclosures. Christopher Paul Fox: Abbvie, Roche, GIlead Takeda Consultancy, research funding, travel to scientific conferences. Celgene/BMS, Janssen Consultancy, travel to scientific conferences. AstraZeneca, Gilead, GenMab, Incyte: Consultancy. Wendy Osborne: Roche, takeda, Pfizer, Servier, Kite Gilead, MSD, Novartis, Beigene, Astra Zeneca, Syneos, Autolus.

Compliance with Ethics Guidelines. This article does not contain any studies with human participants or animals performed by any of the authors.

Peer Review. Please note, contrary to the journal's standard single-blind peer review process, as a podcast this article underwent review by a member of the journal's Editorial Board.
Open Access. This article is licensed under a Creative Commons Attribution-NonCommercial 4.0 International License, which permits any non-commercial use, sharing, adaptation, distribution and reproduction in any medium or format, as long as you give appropriate credit to the original author(s) and the source, provide a link to the Creative Commons licence, and indicate if changes were made. The images or other third party material in this article are included in the article's Creative Commons licence, unless indicated otherwise in a credit line to the material. If material is not included in the article's Creative Commons licence and your intended use is not permitted by statutory regulation or exceeds the permitted use, you will need to obtain permission directly from the copyright holder. To view a copy of this licence, visit http://creativecommons.org/licenses/by$\mathrm{nc} / 4.0 /$.

\section{REFERENCES}

1. Jacobson C, Chavez JC, Sehgal AR, et al. Primary analysis of Zuma-5: a phase 2 study of axicabtagene ciloleucel (Axi-Cel) in patients with relapsed/refractory $(\mathrm{R} / \mathrm{R})$ indolent non-Hodgkin lymphoma (iNHL). https://ash.confex.com/ash/2020/webprogram/ Paper136834.html.

2. Fowler NH, Dickinson M, Dreyling M, et al. Efficacy and safety of tisagenlecleucel in adult patients with relapsed/refractory follicular lymphoma: interim analysis of the phase 2 Elara trial. ASH 2020 conference. 2020. https://ash.confex.com/ash/2020/ webprogram/Paper138983.html.

3. Ramakrishnan A, Ardeshna KM, Batlevi CL, et al. Phase 1 Alexander study of AUTO3, the first CD19/22 dual targeting CAR $\mathrm{T}$ cell therapy, with pembrolizumab in patients with relapsed/refractory $(\mathrm{r} / \mathrm{r})$ DLBCL. ASH 2020 conference. 2020. https://ash. confex.com/ash/2020/webprogram/Paper133960. html.

4. Borchmann P, Jühling A, Gödel P, et al. Phase I trial of MB-CART2019.1, a novel CD20 and CD19 targeting tandem chimeric antigen receptor, in patients with relapsed or refractory B-cell non-hodgkin lymphoma. ASH 2020 conference. 2020. https://ash.confex.com/ ash/2020/webprogram/Paper136067.html.

5. Cao Y, Xiao Y, Wang N, et al. D19/CD22 CAR-T cell cocktail therapy following autologous 
transplantation in patients with relapsed/refractory B-cell lymphomas. ASH 2020 conference. 2020. https://ash.confex.com/ash/2020/webprogram/ Paper140308.html.
6. Neelapu SS, Dickinson S, Ulrickson ML, et al. Interim analysis of ZUMA-12: a phase 2 study of axicabtagene ciloleucel (Axi-Cel) as first-line therapy in patients (pts) with high-risk large B cell lymphoma (LBCL). ASH 2020 conference. 2020. https://ash.confex.com/ ash/2020/webprogram/Paper134449.html. 